\title{
INCIDÊNCIA DE FRATURAS EM CÃES E GATOS DA CIDADE DE MAPUTO (MOÇAMBIQUE) NO PERÍODO DE 1998-2008
}

\author{
ATANASIO SERAFIM VIDANE ${ }^{1,3}$, MARIO ZeFanias JoAo Elias ${ }^{2}$, Jose MANUEl Mota

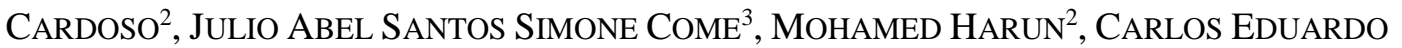 \\ $\mathrm{AMBRÓSIO}^{4}$
${ }^{1}$ Doutorando no Programa de Anatomia dos Animais Domésticos e Silvestres, FMVZ/USP - Brasil.
${ }^{2}$ Professor Associado Faculdade de Medicina Veterinária UEM- Moçambique
${ }^{3}$ Professor Assistente Faculdade de Medicina Veterinária UEM- Moçambique
${ }^{4}$ Professor Associado Faculdade de Zootecnia e Engenharia de Alimentos, Universidade de São Paulo, São Paulo, SP, Brasil - ceambrosio@usp.br

RESUMO

\begin{abstract}
As fraturas representam o principal problema ortopédico na clínica de pequenos animais. O estudo de 333 casos em cães, e 37 em gatos diagnosticados nas principais unidades de atendimento veterinário da cidade de Maputo no período de 1998 a 2008 foi levado a cabo para analisar a frequência das fraturas. A maior incidência de fraturas foi registrada em animais com idade igual ou inferior a 2 anos $(55,68 \%)$. Em ambas as espécies, os machos $(58,38 \%)$ estiveram mais envolvidos em fraturas que as fêmeas. $\mathrm{O}$
\end{abstract}

esqueleto apendicular foi o mais acometido com 91,59\% (cães) e 75,66\% (gatos) do total de fraturas, sendo o fêmur $[35,74 \%$ (cães) e $35,14 \%$ (gatos)] singularmente o mais envolvido em todas as espécies seguido pela tíbia e fíbula. Os atropelamentos por veículos motorizados foram responsáveis pela maior parte das fraturas, contudo, a frequência por agressão humana é bastante preocupante. Não há evidência da distribuição sazonal dos casos registrados.

PALAVRAS-CHAVE: cães; fraturas; gatos; radiografias; traumatismos

\section{INCIDENCE OF FRACTURES IN THE DOGS AND CATS IN MAPUTO (MOZAMBIQUE) BETWEEN 1998 AND 2008}

\section{ABSTRACT}

The fractures represent an important health hazard in orthopedic practices of small animals. A Survey of 333 canine and 37 feline fractures diagnosed at main veterinary hospitals in Maputo between January 1998 and December 2008 was carried out aiming to analyze the frequency of fractures. The highest incidence was recorded in young animals $(55.68 \%)$ under two years of age. The males were more involved $(58.38 \%)$ than the females. The appendicular skeleton showed more frequency of fractures $91.59 \%$ (dogs) and $75.66 \%$ (cats), and among various bones, the femur $[35.74 \%$ (dogs) and $35.14 \%$ (cats)] was more affected followed by tibia and fibula in both species. Most fractures were caused by vehicle accidents; however, the frequency by human aggression is quite worrying. There was no evidence of seasonal distribution of the recorded fractures

KEYWORDS: : cats; dogs; fractures; radiographs; traumatism. 


\section{INTRODUÇÃO}

Diversos casos de traumas em animais domésticos são atendidos e registrados nas clínicas veterinárias ${ }^{1-4}$, sendo que as fraturas representam o principal problema ortopédico na clínica de pequenos animais ${ }^{5},{ }^{6}$. Conhecendo os tipos e a frequência das fraturas nos animais domésticos, os investigadores e profissionais da área ortopédica e de fisioterapia veterinária podem direcionar sua atenção para o aperfeiçoamento de técnicas de fixação, correção e estabilização de fraturas com maior incidência, aumentando a eficiência no seu tratamento e reparação ${ }^{6}$. $O$ presente estudo retrospectivo foi levado a cabo para analisar a frequência das fraturas em cães e gatos tratados nas principais unidades de atendimento veterinário da cidade de Maputo (Moçambique). A importância desta análise reside no maior número de casos reportados, quantidade não observada em nenhum estudo do gênero em Moçambique até ao presente momento, possibilitando maior confiabilidade dos resultados obtidos.

\section{MATERIAL E MÉTODOS}

Foram analisadas as radiografias de fraturas e suas respetivas fichas de registro dos casos atendidos e tratados no período de Janeiro de 1998 a Dezembro de 2008 em três unidades de atendimento veterinário da cidade de Maputo-Moçambique, nomeadamente: Hospital Escolar Veterinário-
Universidade Eduardo Mondlane (HEV-UEM), clínica Veterinários Associados Ltda.(VAL) e clínica IBA-VET. As fichas de registro e as radiografias foram agrupadas por ano de ocorrência das fraturas. Nas radiografias foram analisadas a localização e o tipo de fratura; das fichas de registro foram extraídas informações referentes à espécie, idade e sexo do animal, causa da fratura e período de ocorrência ao longo do ano. Os resultados obtidos foram expressos em tabelas contendo os números absolutos e as frequências das observações. A comparação entre as frequências foi feita no programa estatístico "EpiCalc 2000" a um nível de significância de 5\%.

\section{RESULTADOS E DISCUSSÃO}

Um total de 393 casos de fraturas foi registrado nas unidades de atendimento veterinário da cidade de Maputo durante o período em análise. Este registro não reflete o total de ocorrências de fraturas na cidade de Maputo. Por motivos diversos, muitos casos de fraturas em animais domésticos não são encaminhados ao atendimento médico adequado. Do total dos casos reportados, os cães apresentaram maior frequência de fraturas $(84,7 \%)$ em relação aos gatos $(9,4 \%)$ (Figura 1). Esse resultado se deve ao fato de terem sido atendidos mais cães que as demais espécies. A maior entrada dos cães com casos de fraturas é consequência do fato de os cães serem animais de estimação mais populosos em Moçambique.

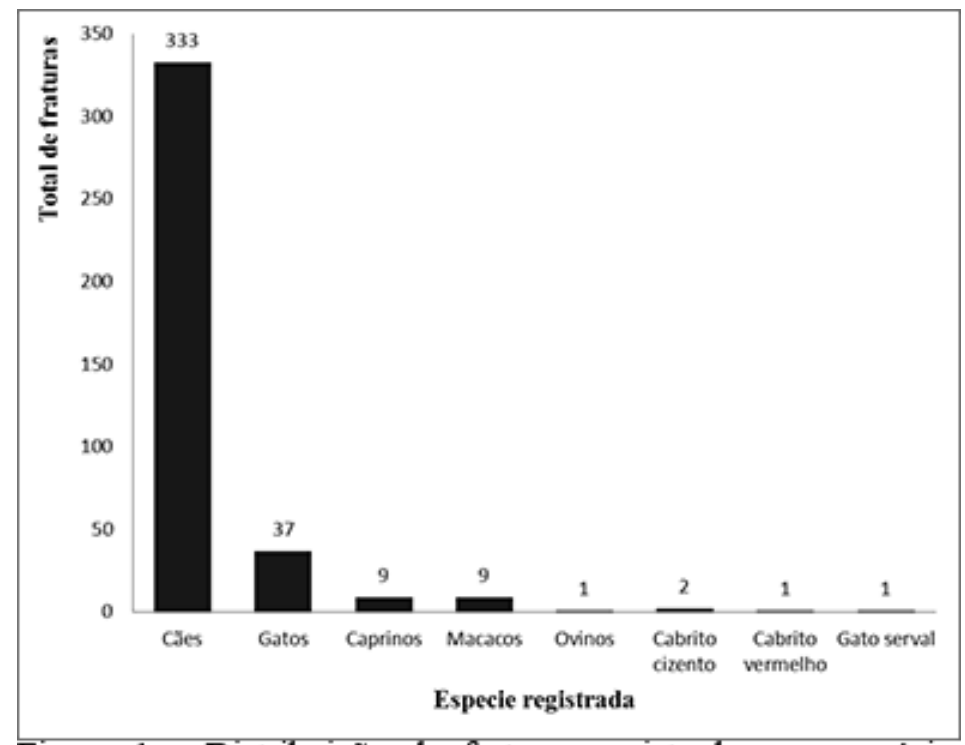

Figura 1 - Distribuição de fraturas registradas por espécie, atendida nas principais unidades de tratamento na Cidade de Maputo (Moçambique) no período de Janeiro de 1998 a Dezembro de 2008. 
Os machos $(58,3 \%)$ estiveram mais envolvidos em fraturas que as fêmeas, à semelhança dos achados reportados em cães e gatos ${ }^{1,3,4,6-9}$ (Tabela 1). A natureza agressiva e o comportamento ativo especialmente na época de maior atividade reprodutiva justificam a maior vulnerabilidade dos machos a fraturas.

Tabela 1 - Números absolutos e frequências (\%) de fraturas, por espécie e sexo, de 393 casos inventariados em clínicas veterinárias da cidade de Maputo no período de Janeiro de 1998 a Dezembro de 2008

\begin{tabular}{ccccccc}
\hline Sexo & $\begin{array}{c}\text { Cães } \\
(\%)\end{array}$ & $\begin{array}{c}\text { Gatos } \\
(\%)\end{array}$ & $\begin{array}{c}\text { Macacos } \\
(\%)\end{array}$ & $\begin{array}{c}\text { Caprinos } \\
(\%)\end{array}$ & Outras spp(\%) & Total (\%) \\
\hline Macho & $193(58,0)$ & $23(62,2)$ & $5(55,6)$ & $5(55,6)$ & $3(60,0)$ & $229(58,3) \mathrm{a}$ \\
\hline Fêmea & $140(42,0)$ & $14(37,8)$ & $4(44,4)$ & $4(44,4)$ & $2(40,0)$ & $164(41,7) \mathrm{b}$ \\
\hline Total $(\%)$ & $333(84,7)$ & $37(9,4)$ & $9(2,3)$ & $9(2,3)$ & $5(1,3)$ & $393(100)$ \\
\hline
\end{tabular}

No que diz respeito à idade, foi registada maior incidência de fraturas em animais com idade igual ou inferior a dois anos $(55,68 \%)$, à semelhança dos achados de estudos anteriores em cães ${ }^{6}$ e gatos ${ }^{8}$. A maior frequência de fraturas em animais jovens pode ser sustentada pelo fato destes apresentarem ossos frágeis, ainda em crescimento, e a falta de habilidade para evitar traumas.

Os atropelamentos por veículos motorizados foram responsáveis pela maior parte das fraturas em cães $(22,22 \%)$. A predominância de cães atropelados foi igualmente reportada por Adeyanju et al. ${ }^{1}$, Fighera et al. ${ }^{3}$, Kolata et al. ${ }^{7}$ e Mendes et al. ${ }^{10}$. A maior frequência de atropelamentos em canídeos se deve ao fato de as disposições legais em vigor no país não serem cumpridas, no que diz respeito às medidas de contenção e proteção dos animais que utilizam os espaços públicos. Além disso, nos últimos anos tem-se registrado um crescente aumento de veículos motorizados na cidade de Maputo, o que vem a agravar esta ocorrência. Os atropelamentos também podem ser correlacionados à maior frequência de fraturas em machos. A procura de fêmeas em cio e a briga entre os machos sujeita esses animais a maior vulnerabilidade para atropelamento por veículos motorizados.

Em gatos, a maior parte de fraturas foi causada por quedas diversas $(10,8 \%)$. As causas de fraturas em felinos domésticos divergem das reportadas por Adeyanju et al. ${ }^{1}$, que constataram como causa principal atropelamentos.

Embora a maior parte dos casos registrados tenha causas desconhecidas, a frequência de fraturas por agressão humana $(4,32 \%)$, para ambas as espécies, reflete a necessidade de mudança de atitude das pessoas em relação aos animais de estimação bem como a intensificação dos cuidados e proteção dos mesmos.

A distribuição das frequências das fraturas ao longo do ano é pouco variável. Houve uma tendência de maior concentração de fraturas nos meses de Abril, Julho e Dezembro, porém esta variação não é significativa (Figura 2). A explicação para esta tendência é desconhecida. Em estudos anteriores envolvendo cães e gatos ${ }^{1,11}$, foi reportada uma maior incidência de fraturas nas épocas de maior atividade reprodutiva (Fevereiro-Abril e Setembro-Novembro). Os estudos de Elias $^{2}$ não observaram clara evidência da distribuição sazonal de fraturas e não tiveram explicação para os seus achados. É importante ressaltar o fato de Moçambique ser um país de clima tropical quente e húmido, com poucas variações climáticas e da intensidade solar ao longo do ano, o que pode mascarar a sazonalidade reprodutiva dos animais domésticos. 


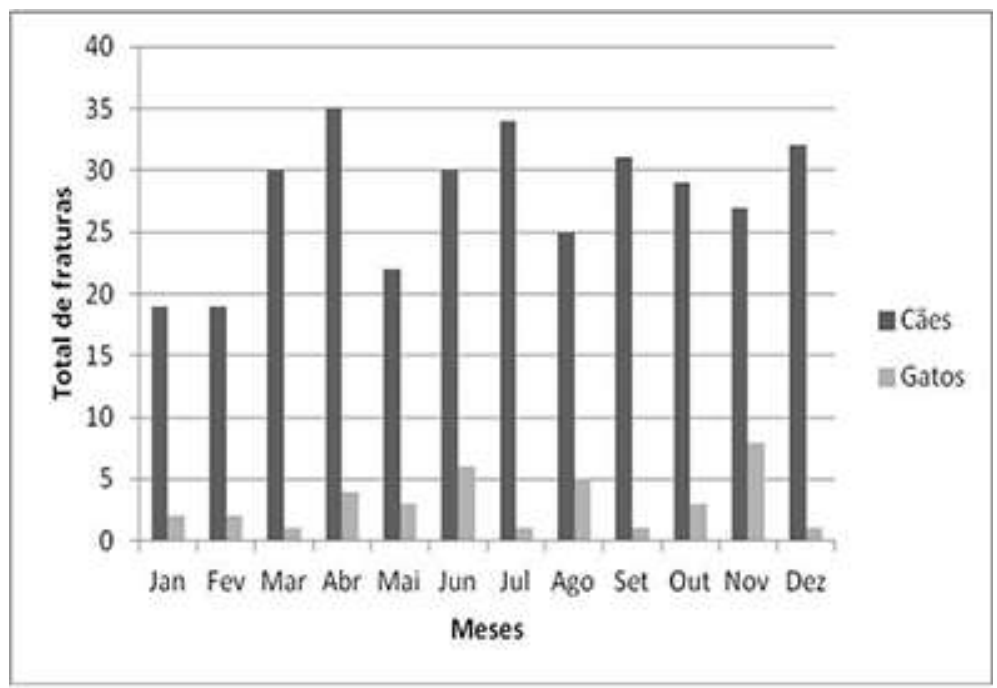

Figura 2 - Distribuição de fraturas em cães e gatos ao longo do ano na cidade de Maputo no periodo de 1998-2008.

Neste estudo, o esqueleto apendicular foi o mais acometido com $91,59 \%$ (cães) e 75,66\% (gatos) do total das fraturas. Destas, o fêmur foi singularmente o mais envolvido em todas as espécies, contabilizando $35,74 \%$ (cães) e 35,14 (gatos), seguido pela tíbia e fíbula com $21,92 \%$ (cães) e 18,92\% (gatos). Esses achados corroboram com os achados reportados em cães e gatos por Adeyanju et al. ${ }^{1}$; Elias ${ }^{2}$; Fighera et al. ${ }^{3}$; Shiju Simon et al. ${ }^{6}$; Wong ${ }^{8}$; Alcantara \& Stead ${ }^{12}$. As fraturas do fêmur e do esqueleto crural são geralmente relacionadas aos atropelamentos e a traumas por quedas diversas. Apesar da alta cobertura muscular envolvendo o fêmur, esta não contribui para proteção contra fraturas quando ocorre este tipo de acidentes.

No membro torácico, as fraturas do rádio e ulna ocorreram com maior frequência em cães $(12,01 \%)$, seguidas das fraturas do úmero $(6,91 \%)$. Em estudos anteriores de fraturas diafisárias envolvendo cães e gatos ${ }^{1}$, não foi reportado um padrão claro da incidência das fraturas no membro torácico.

Em relação ao esqueleto axial, foi registrada uma maior frequência das fraturas mandibulares em cães $(2,10 \%)$ e gatos $(10,81 \%)$, seguidos das da cauda e da maxila. Também foram reportados apenas dois casos de fraturas do osso peniano em cães. Em geral as fraturas do esqueleto axial e visceral são raras e pouco estudadas. Esses casos estão associados a lutas e mordeduras entre animais bem como aos casos de agressão humana.

\section{CONCLUSÕES}

Os casos de fraturas em animais tratados nas clínicas da cidade de Maputo envolvem majoritariamente cães e gatos, com maior frequência em machos e animais jovens. As fraturas do fêmur são as que mais se destacam geralmente associadas a casos de atropelamentos e quedas diversas. A distribuição anual das fraturas tende a ser homogênea sem muita relação com a sazonalidade reprodutiva destas espécies.

\section{AGRADECIMENTOS}

Aos representantes do Hospital Escolar Veterinário, Clínica VAL e IBA-VET (Maputo, Moçambique) pela disponibilização dos dados.

\section{REFERÊNCIAS}

1. Adeyanju, JB, Garba M, Elijah A. Diaphyseal fractures in small animals: management and complications. Tropical Veterinary., v.6, p.95-103, 1988.

2. Elias MZJ. Frequência de ossos fracturados nos membros de carnívoros domésticos atendidos no hospital escolar veterinário (1969-1995). O Agrário-revista científica e de divulgação., v.20, p.4-7, 2000.

3. Fighera RA, Silva MC, Souza TM, Brum JS, Kommers GD, Graça DL, Irigoyen LF, Barros CSL. Aspectos patológicos de 155 casos fatais de cães atropelados por veículos automotivos. Ciência Rural., v.38, n.5, p.13751380, 2008. 
4. Kumar K, Mogha IV, Aithal HP, Kinjavdekar, P, 9. Ferriono CRA Schmaedecke A, Unruh SM, Pinto Amarpal, Singh GR, Pawde AM, Kushwaha RB. ACBCF, Sterman FA, Giglio RF. Estudo retrospectivo de Occurrence and Pattern of Long Bone Fractures in radiografias com fraturas do rádio e ulna em cães. Braz. J. Growing Dogs with Normal and Osteopenic Bones. J. Vet. Med. A., v.54, p.484-490, 2007.

5. Kolata RJ. Trauma in dogs and cats: an overview. Vet Clin North Am Small Anim Pract., v.10, n.3, p.515-22, 1980 .

vet. Res. anim. Sci., v.44, p.122-124, 2007.

10. Mendes DS, Arias MVB. Traumatismo da medula espinhal em cães e gatos: estudo prospectivo de 57 casos. Pesquisa Veterinária Brasileira, v.32, n.12, p.1304-1312, 2012.

6. Shiju SM, Ganesh R, Ayyappan S, Rao GD, Kumar RS, Kundave VR, Das BS. Incidences of pelvic limb fractures in dogs: A survey of 478 cases. Veterinary World., v.3, n.3, p.120-121, 2010.

7. Kolata RJ, Kraut NH, Johnson HH. Patterns of trauma in urban dogs and cats: A study of 1000 cases. Journal of the American Veterinary Association., v.164, p.499-502, 1974.

8. Wong WT. A survey of fractures in the dog and cat in Malaysia. Veterinary Record., v.115, p.273-274, 1984.

11. Lucas SS, Schossler JEW, Cony AV, Alievi MM. Fraturas distais de fêmur em cães e Gatos. Revisão de 55 casos. Revista da FZVA Uruguaiana., v.7/8, n.1, p.75-83, 2000-01.

12. Alcantara PJ, Stead AC. Fractures of the distal femur in the dog and cat. Journal Small Animal Practice., v.16, p.649-659, 1975.

13. Costa RC, Schossler JEW. Tratamentos de fraturas do rádio e da ulna em cães e gatos: revisão. Archives of Veterinary Science., v.7, n.1, p.89-98, 2002.

Protocolado em: 23 maio 2013. Aceito em: 04 set. 2014-09-04 